\title{
Modified ileal conduit intracorporeally accomplished following laparoscopic radical cystectomy with enhanced recovery protocols: experience with 48 cases
}

\author{
Houyi Wei ${ }^{1}$, Mingshuai Wang ${ }^{1}$, Wahafu Wasilijiang ${ }^{2}$, Wei Wang ${ }^{1}$, Xing Guan ${ }^{1}$, Xiaoguang Zhou ${ }^{1}$, \\ Liming Song ${ }^{1}$, Nianzeng $\mathrm{Xing}^{2}$, Yinong $\mathrm{Niu}^{1}{ }^{\wedge}$ \\ ${ }^{1}$ Institute of Urology, Capital Medical University, Department of Urology, Beijing Chao-Yang Hospital, Capital Medical University, Beijing, China; \\ ${ }^{2}$ Department of Urology, National Cancer Center/National Clinical Research Center for Cancer/Cancer Hospital, Chinese Academy of Medical \\ Sciences and Peking Union Medical College, Beijing, China \\ Contributions: (I) Conception and design: All authors; (II) Administrative support: N Xing, Y Niu; (III) Provision of study materials or patients: L \\ Song, N Xing, Y Niu; (IV) Collection and assembly of data: All authors; (V) Data analysis and interpretation: All authors; (VI) Manuscript writing: \\ All authors; (VII) Final approval of manuscript: All authors. \\ Correspondence to: Yinong Niu, MD. 8 Gongren Tiyuchanag Nanlu, Chaoyang District, Beijing, China. Email: 18601020160@163.com.
}

Background: This study introduces the results of laparoscopic radical cystectomy with modified intracorporeal ileal conduit (mICIC), which was accompanied by enhanced recovery after surgery (ERAS) protocols.

Methods: From March 2014 to June 2020, 48 patients underwent mICIC. Patients were divided into ERAS ( $\mathrm{n}=17$ ) and non-ERAS groups $(\mathrm{n}=31)$. Baseline and perioperative variables were analyzed. The primary outcome was 90-day complications. Secondary outcomes were operative time, length of stay, two-year overall survival, cancer-specific survival, and disease-free survival.

Results: Forty-eight patients underwent intracorporeal ileal conduit with no transition to open surgery. Twenty-five patients (52.1\%) experienced at least one complication, including 22 minor cases (45.8\%) and three major cases $(6.2 \%)$. The median operative time, urinary diversion time, estimated blood loss, and length of stay were $320 \mathrm{~min}, 135 \mathrm{~min}, 200 \mathrm{~mL}$, and 10.5 days, respectively. The median time to flatus and normal diet were two days and three days, respectively. A comparison between ERAS and non-ERAS groups indicated that ERAS implementation was associated with less complications $(29.4 \%$ vs. $64.5 \%$, $\mathrm{P}=0.018)$, faster time to flatus $(2$ vs. 3 days, $\mathrm{P}=0.016)$ and liquid $\operatorname{diet}(2$ vs. 4 days, $\mathrm{P}<0.001)$. The results of hydronephrosis and compromised renal function showed no difference between the preoperative period and six months after surgery. The mean follow-up time was 25.4 months, and the two-year overall survival, cancer-specific survival, and disease-free survival rates were $61.3 \%, 73.2 \%$, and $58.4 \%$, respectively.

Conclusions: The complication rate and operative time of the mICIC were acceptable. Clinical outcomes can be optimized with ERAS pathway.

Keywords: Bladder cancer; laparoscopic radical cystectomy (LRC); modified intracorporeal ileal conduit; enhanced recovery after surgery (ERAS)

Submitted Dec 23, 2020. Accepted for publication Feb 18, 2021.

doi: $10.21037 /$ tau-20-1515

View this article at: http://dx.doi.org/10.21037/tau-20-1515

$\wedge$ ORCID: 0000-0003-0870-4056. 


\section{Introduction}

Radical cystectomy (RC) and pelvic lymph node dissection is used to treat muscle-invasive bladder cancer and high-risk and recurrent non-muscle-invasive bladder cancer. Recently, significant progress has been made in minimally invasive surgery, including laparoscopic radical cystectomy (LRC) and robot-assisted radical cystectomy (RARC). When compared with open RC, LRC and RARC result in better clinical outcomes, while still achieving equivalent pathologic and oncologic results (1-3). Extracorporeal urinary diversion (ECUD) is easy to construct and is accepted by many urologists as a common treatment. However, patients may benefit more from intracorporeal urinary diversion (ICUD) due to reduced bowel exposure and decreased risk of fluid imbalance (4).

Enhanced recovery after surgery (ERAS) protocols, first initiated in colorectal surgeries (5), were recently introduced for urologic surgeries (6). Increasing evidence from gastrointestinal and colorectal surgeries indicated that the application of ERAS protocols could improve postoperative recovery and reduce the time to flatus, defecation, and functional oral intake $(7,8)$. This study details the experience of our modified intracorporeal ileal conduit (mICIC) using the results of 48 cases. We present the following article in accordance with the STROBE reporting checklist (available at http://dx.doi.org/10.21037/tau-20-1515).

\section{Methods}

Between March 2014 and June 2020, 48 consecutive patients with bladder cancer underwent LRC with mICIC. Indications included muscle-invasive bladder cancer, T1G3 bladder cancer, recurrent non-muscle-invasive bladder cancer, or extensive papillary tumors that could not be managed with transurethral resection and intravesical instillation therapy. All patients underwent cystoscopy examination, pelvic magnetic resonance imaging, or enhanced computed tomography before surgery. No distant metastases were found on preoperative examinations. ERAS protocols were introduced in our institution since April 2017, and patients were divided into the ERAS and nonERAS groups. The study was conducted in accordance with the Declaration of Helsinki (as revised in 2013). The study was approved by the Institutional Review Board of Beijing Chaoyang Hospital, Capital Medical University (NO.: 2020-Ke-546) and informed consent for this retrospective analysis was waived.

\section{Data collection}

Baseline characteristics, perioperative results, pathologic results, oncologic outcomes, and follow-up data were evaluated. Complications occurring within 90 days after surgery were recorded and graded according to the Clavien-Dindo system, with a grade of 1-2 defined as a minor complication and a grade of 3-5 defined as a major complication. Severe hypoalbuminemia was defined as a serum albumin level of $<25 \mathrm{~g} / \mathrm{L}$. Hydronephrosis was measured by the separation of the collection system through ultrasonography or computed tomography: mild, $\geq 2$ and $<3 \mathrm{~cm}$; moderate, $\geq 3$ and $<4 \mathrm{~cm}$; and severe, $\geq 4 \mathrm{~cm}$. Compromised renal function was estimated by creatinine clearance rate: normal, $>80 \mathrm{~mL} / \mathrm{min}$; mild, $>50$ and $\leq 80 \mathrm{~mL} / \mathrm{min}$; moderate, $>10$ and $\leq 50 \mathrm{~mL} / \mathrm{min}$; and severe, $<10 \mathrm{~mL} / \mathrm{min}$. Two-year overall survival (OS), cancerspecific survival (CSS), and disease-free survival (DFS) were calculated. OS was defined as the duration from RC to death from any cause. CSS was defined as the duration from RC to death from bladder cancer. DFS was defined as the duration from RC to recurrence (local, regional, and/or distant) or death from any cause.

\section{Surgical technique}

After general anesthesia, the patient's limbs were tied to the operating table. A nasogastric tube and urethral catheter were inserted separately. The patient was placed in a steep Trendelenburg position $\left(30^{\circ}\right)$.

The trocar was configured using a six-port peritoneal approach. After pneumoperitoneum was achieved, a $10-\mathrm{mm}$ camera port was placed $1.5 \mathrm{~cm}$ supraumbilically. A $0^{\circ}$ flexible camera was inserted, and the following trocar placements were performed under direct visual control. Two $12-\mathrm{mm}$ ports were placed on the bilateral rectus line at the level of the umbilicus. Two 5 -mm ports were placed $3 \mathrm{~cm}$ from the interior of the anterior superior iliac spine on each side. Another $12-\mathrm{mm}$ port was placed $1 \mathrm{~cm}$ cranial to the symphysis pubis to insert the Endo-GIA ${ }^{\mathrm{TM}}$ stapler (Medtronic, Minneapolis, MN, USA) and another laparoscopic light source. At the end of the surgery, the suprapubic incision was extended to $4-5 \mathrm{~cm}$ to facilitate specimen removal.

Standard pelvic lymph node dissection was performed. Extended lymph node dissection was performed when needed, and involved removal of nodal tissue from the internal iliac, external iliac, presacral, obturator fossa nodes, 


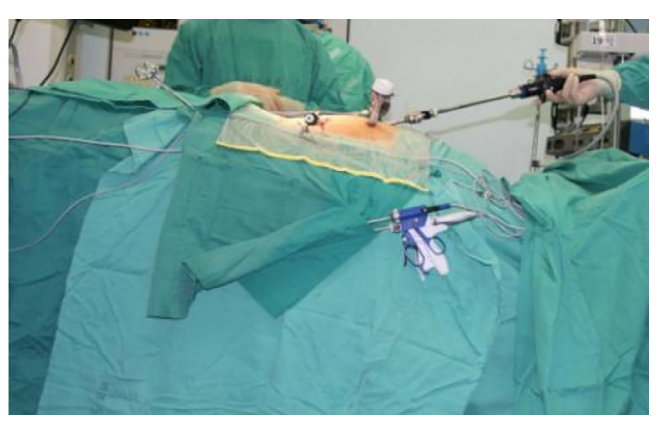

Figure 1 Trendelenburg position.

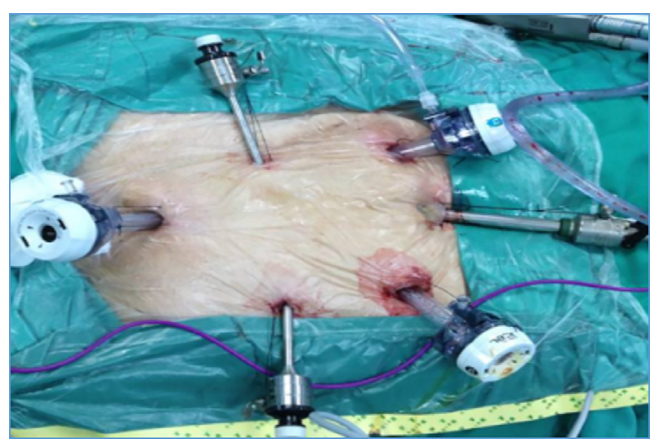

Figure 2 Trocar configuration.

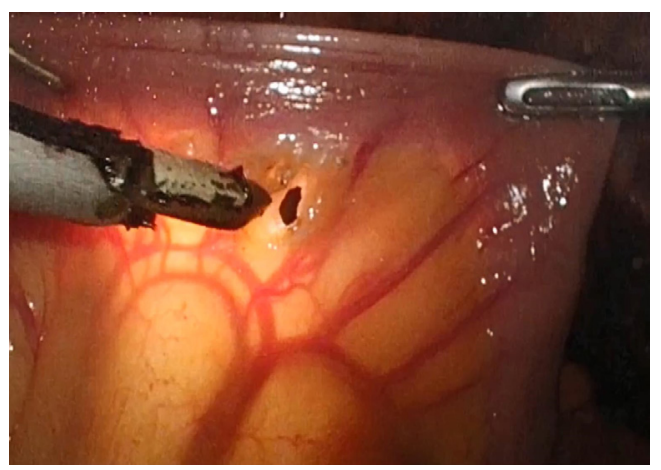

Figure 3 The mesentery was transilluminated.

and cranially up to the aortic bifurcation. The lateral border was the genitofemoral nerve, the medial border was the ureter, and the inferior border was the Cloquet lymph node and circumflex iliac vein.

Urinary diversion was performed as follows. The left ureter was brought over the great vessels to the right side. Using a linear stapler, a $15-\mathrm{cm}$ terminal ileum was isolated $15 \mathrm{~cm}$ from the ileocecal valve, during which the mesentery was transilluminated to avoid vascular arcade injury and preserve blood supply to the involved intestine. Continuity of the ileum was restored by endto-end ileoileal anastomosis using a linear stapler. The isolated loop was flushed with normal saline containing gentamycin, and two single-J ureteral stents were pulled through the lumen of the loop. A circular incision was made at the stoma site, and an extraperitoneal tunnel was bluntly created with the index finger. Through the tunnel, the loop and stents were pulled out and fixed on the abdominal wall. The distal end of each ureter was spatulated with a $1.5-\mathrm{cm}$ lengthwise incision. The entrance of the efferent loop was virtually divided into two semi-lumens by a midline. Instead of uniting the two ureters by suturing their medial borders, each ureter was directly and individually anastomosed to the efferent loop. The posterior wall of the left ureter was anastomosed to the posterior wall of the left semi-lumen using running sutures, with the lower end of the ureteral incision approaching the midline and the upper end approaching the semi-lumen's lateral border. The same procedure was repeated on the right ureter and the right semi-lumen (9). The anterior walls of the ureters and semi-lumens were anastomosed after two single-J ureteral stents were inserted into the bilateral ureters. Finally, the distal ureters, efferent loop, and ureterointestinal anastomosis were fully retroperitonealized by closing the rent of the retroperitoneum.

Briefly, the key steps of the mICIC were: the mesentery was transilluminated when isolating the terminal ileum; with the conduit fixed in the extraperitoneal tunnel, a novel end-to-end reflux ureterointestinal anastomosis was performed; and the rent of the retroperitoneum was closed using running sutures at the end of surgery. These key steps are presented in Figures 1-10.

\section{Perioperative management (ERAS protocols)}

Preoperative: Thromboembolic prophylaxis (compression stockings and low molecular weight heparin) was administered before surgery. A laxative was administered and the normal diet was changed to a liquid food diet 1 day preoperatively. Liquid food was allowed until 6 hours preoperatively. Potable water and oral carbohydrate loading were allowed up to 2 hours before surgery; after that, any oral intake was abandoned.

Intraoperative: Antibiotics were administered 0.5 hours preoperatively; a second dose was added if the operative time exceeded 3 hours. The intravenous fluid 


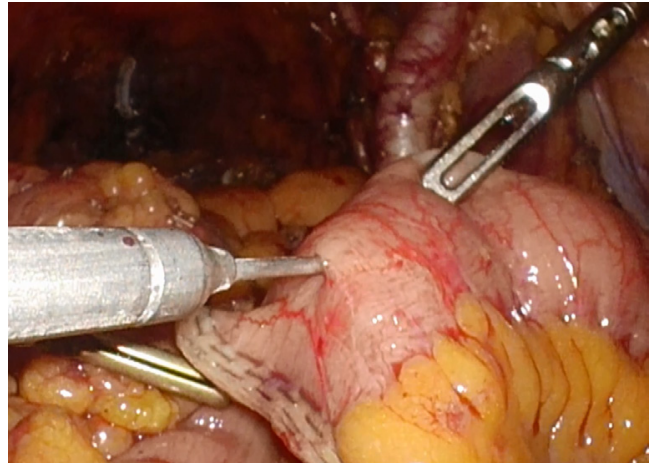

Figure 4 Normal saline containing gentamycin was injected into the efferent loop.

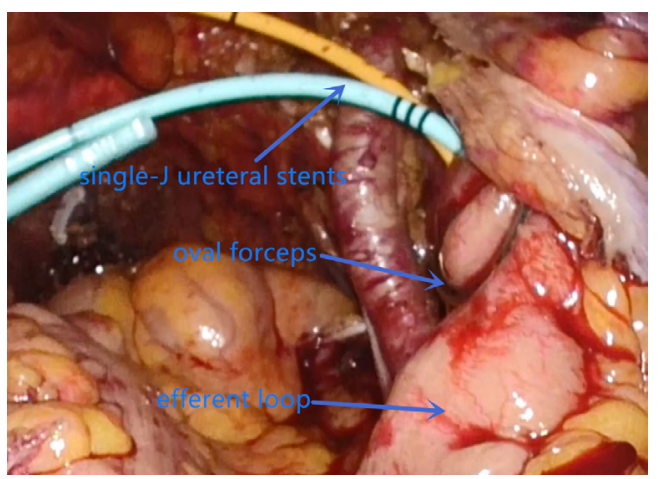

Figure 5 The efferent loop and the single-J ureteral stents were pulled out (the oval forceps was inserted through the extraperitoneal tunnel).

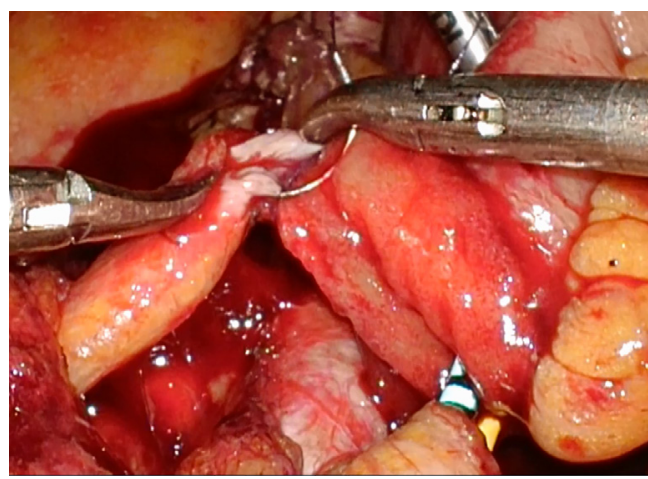

Figure 6 The posterior walls of spatulated ureters were sutured to the proximal end of the efferent loop in running fashion.

supplementation was limited and targeted to losses. Intraoperative hypothermia was avoided by blowing warm air over the body. The nasogastric tube was removed

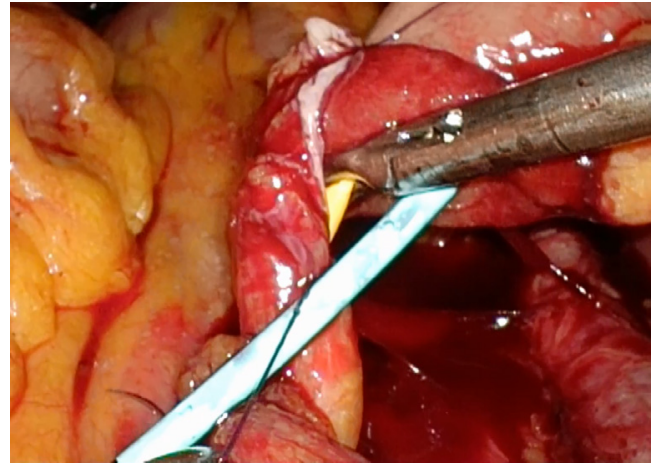

Figure 7 Two single-J ureteral stents were inserted into bilateral ureters respectively.

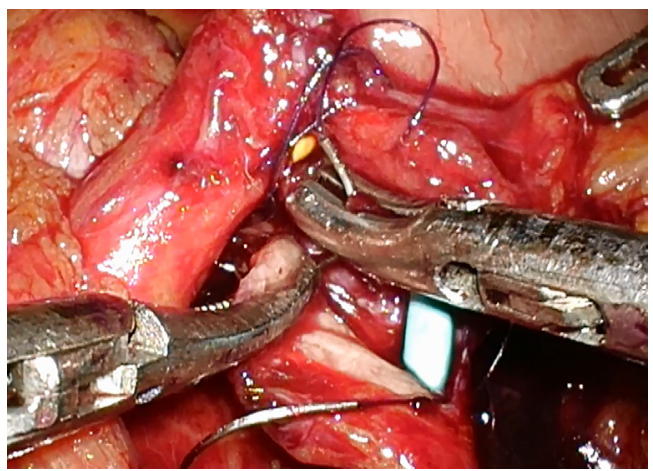

Figure 8 The anterior walls of spatulated ureters and the efferent loop were sutured in running fashion.

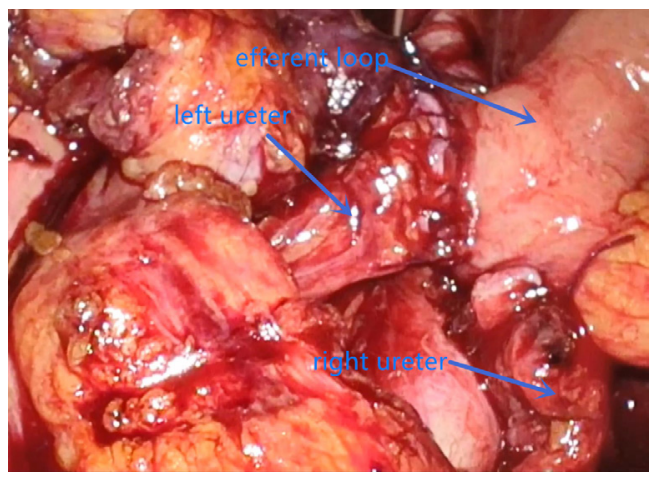

Figure 9 The ureters and the efferent loop were anastomosed.

immediately after awakening.

Postoperative: Non-steroidal anti-inflammatory drugs, rather than opioids, were used to relieve pain. Early mobilization was encouraged, which included getting up slowly 4 hours postoperatively and attempting to get out of 


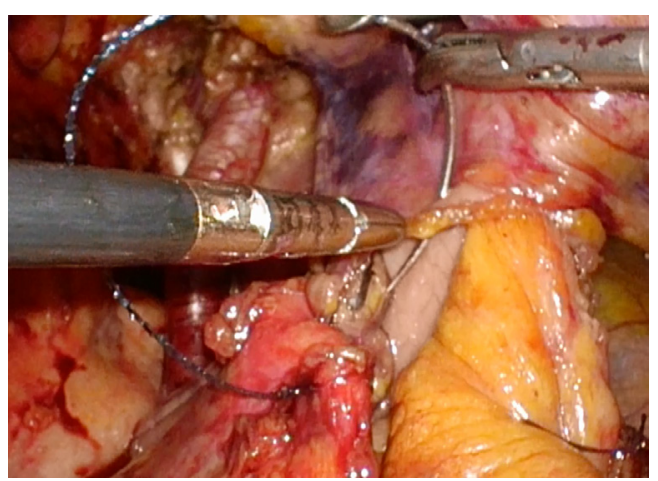

Figure 10 The rent of the retroperitoneum was closed.

bed 6 hours postoperatively. Chewing gum was encouraged beginning 4 hours after surgery $(30 \mathrm{~min}$ each time, 3 times a day until passing flatus). Oral fluid intake was administered early $(50 \mathrm{~mL} / \mathrm{h}$ of potable water 6 hours after surgery and increased to $100 \mathrm{~mL} / \mathrm{h}$ on postoperative day 1). After passing flatus, liquid food was resumed and gradually converted to a normal diet.

\section{Statistical analysis}

SPSS v.26 (IBM Corp., Armonk, NY, USA) was used for statistical analysis. Normally distributed continuous variables are expressed as mean values with standard deviations, and were compared using Student's t-test. Nonnormally distributed continuous variables are expressed as median and interquartile ranges, and were compared using the Mann-Whitney U-test. Categorical variables were compared using the Chi-square test or Fisher's exact test. The two-year OS, CSS, and DFS were assessed by the Kaplan-Meier estimator.

\section{Results}

The baseline characteristics and perioperative results are presented in Table 1. Surgery was accomplished intracorporeally with no transition to open surgery in all 48 cases. The median operative time, urinary diversion time, estimated blood loss, and length of stay were $320 \mathrm{~min}$, $135 \mathrm{~min}, 200 \mathrm{~mL}$, and 10.5 days, respectively. The median time to flatus and liquid diet were two and three days, respectively. Six patients $(12.5 \%)$ required intraoperative blood transfusion, and the mean estimated blood loss was $433.3 \mathrm{~mL}$. When comparing the ERAS and non-ERAS groups, the differences in the male/female ratio $(\mathrm{P}=0.018)$ and history of smoking $(\mathrm{P}=0.034)$ were statistically significant. The ERAS group also had a significantly shorter time to flatus ( 2 vs. 3 days, $\mathrm{P}=0.016)$ and liquid diet ( $2 v s$. 4 days, $\mathrm{P}<0.001)$.

Ninety-day complications are presented in Table 2. Twenty-five patients $(52.1 \%)$ experienced at least one complication, including 22 minor cases $(45.8 \%)$ and three major cases $(6.2 \%)$. Severe hypoalbuminemia and emesis were the most common complications. A comparison between the ERAS and non-ERAS groups indicated that ERAS implementation was associated with fewer complications (29.4\% vs. $64.5 \%, \mathrm{P}=0.018)$. Thirteen non-ERAS patients $(41.9 \%)$ experienced severe hypoalbuminemia; none of the ERAS patients experienced this complication. Minor (29.4\% vs. $54.8 \%$, $\mathrm{P}=0.132)$ and Clavien-Dindo grade 1 (17.6\% vs. 48.4\%, $\mathrm{P}=0.073)$ complication rates also tended to be lower in the ERAS group, although the differences were not statistically significant. Three patients in the non-ERAS group experienced major complications. One patient, who developed acute non-ST segment elevation myocardial infarction postoperatively, was transferred to the intensive care unit and received conservative treatment. One patient died on postoperative day 11 due to ventricular fibrillation. One patient was discharged two weeks after surgery, but had severe pneumonia at home and died due to multiple organ failure four weeks postoperatively.

Pathologic results are presented in Table 3. Mean lymph node yield was $17.6 \pm 9.4$ for the whole cohort, $13.9 \pm 6.5$ for the ERAS group, and $19.5 \pm 10.2$ for the non-ERAS group $(\mathrm{P}=0.052)$. Positive surgical margin (PSM) was identified in four patients $(8.3 \%)$; three had pT3 disease and one had pT4 disease.

The morphology and function of the upper urinary tract of 36 patients were evaluated six months after surgery (Table 4). Of these, 33 patients $(91.7 \%)$ developed no or mild hydronephrosis, three patients $(8.3 \%)$ developed moderate or severe hydronephrosis, 32 patients (88.9\%) had no or mildly compromised renal function, and four patients $(11.1 \%)$ had moderately or severely compromised renal function. A comparison of the pre- and postoperative results showed no significant differences (Table 4).

The mean follow-up time was 25.4 months. Overall, 14 patients died during follow-up (including two patients who died within 30 days after surgery). Of these, five patients died due to bladder cancer and nine patients died due to other diseases (organ failure, cerebral stroke, myocardial infarction, etc.). The two-year OS, CSS, and DFS were 
Table 1 Baseline characteristics and perioperative results of 48 patients

\begin{tabular}{|c|c|c|c|c|}
\hline & Overall $(\mathrm{N}=48)$ & ERAS $(\mathrm{N}=17)$ & non-ERAS $(\mathrm{N}=31)$ & $\mathrm{P}^{*}$ \\
\hline Age (years), mean \pm SD & $63.5 \pm 9.1$ & $63.1 \pm 9.6$ & $64.4 \pm 8.5$ & 0.644 \\
\hline Body mass index $\left(\mathrm{kg} / \mathrm{m}^{2}\right)$, mean $\pm \mathrm{SD}$ & $24.5 \pm 3.3$ & $24.4 \pm 3.1$ & $24.5 \pm 3.8$ & 0.919 \\
\hline aCCI, median (IQR) & $4.0(3.0-5.0)$ & $4.0(3.0-6.5)$ & $3.0(2.0-5.0)$ & 0.155 \\
\hline History of neoadjuvant chemotherapy, n (\%) & $9(18.8)$ & $3(17.6)$ & $6(19.4)$ & 1.000 \\
\hline History of abdominal surgery, $\mathrm{n}(\%)$ & $7(14.6)$ & $3(17.6)$ & $4(12.9)$ & 0.686 \\
\hline History of smoking, n (\%) & $23(47.9)$ & $12(70.6)$ & $11(35.5)$ & 0.034 \\
\hline ASA score, n (\%) & & & & 0.651 \\
\hline Operative time (min), median (IQR) & $320.0(270.0-360.0)$ & $340.0(285.0-400.0)$ & $320.0(265.0-350.0)$ & 0.353 \\
\hline Urinary diversion time (min), median (IQR) & $135.0(93.8-161.2)$ & $163.0(150.0-188.0)$ & $125.5(87.0-135.8)$ & $<0.001$ \\
\hline Estimated blood loss (mL), median (IQR) & $200.0(100.0-300.0)$ & $200.0(100.0-200.0)$ & $200.0(100.0-300.0)$ & 0.480 \\
\hline Intraoperative blood transfusion, $\mathrm{n}(\%)$ & $6(12.5)$ & 0 & $6(19.4)$ & 0.077 \\
\hline ICU stay after surgery, n (\%) & $11(22.9)$ & $6(35.3)$ & $5(16.1)$ & 0.163 \\
\hline Time to flatus (day), median (IQR) & $2.0(2.0-3.0)$ & $2.0(1.0-2.0)$ & $3.0(2.0-4.0)$ & 0.016 \\
\hline Time to liquid diet (day), median (IQR) & $3.0(2.0-4.8)$ & $2.0(1.0-2.0)$ & $4.0(3.0-5.0)$ & $<0.001$ \\
\hline Length of stay (day), median (IQR) & $10.5(8.0-13.0)$ & $8.0(7.0-13.0)$ & $11.0(8.0-14.0)$ & 0.138 \\
\hline
\end{tabular}

${ }^{*}$ comparison between ERAS and non-ERAS group; TURBt: transurethral resection of bladder tumor; TURP: transurethral resection of prostate; ASA: American Society of Anesthesiologists; ICU: intensive care unit.

$61.3 \%, 73.2 \%$, and $58.4 \%$, respectively.

\section{Discussion}

LRC with ICUD, first reported in 2000 (10), is a technically challenging procedure that requires extensive operative experience and sophisticated perioperative management. We performed open RC and LRC with ECUD for over a decade, including extracorporeal ileal conduit and extracorporeal orthotopic neobladder procedures. Since 2014, we began performing LRC with ICUD using a modified operative procedure. ERAS protocols were introduced in our institution since April 2017. In this study, 48 patients underwent LRC with $\mathrm{mICIC}$, together with the ERAS pathway. All patients demonstrated acceptable perioperative, oncologic, and functional outcomes. In comparison to the non-ERAS group, the ERAS group showed optimized clinical outcomes.

Results from the International Robotic Cystectomy Consortium (IRCC) was reported in 2014 (4) and showed that $43 \%$ of patients with ECUD and $35 \%$ of patients with ICUD developed 30-day complications $(\mathrm{P}=0.07)$. The ICUD group had fewer gastrointestinal complications $(\mathrm{P}<0.001)$ and postoperative infections $(\mathrm{P}=0.035)$. In 2018, updated results were reported (11), comprising 1094 ICUD cases (51\%) and 1031 ECUD cases (49\%). The proportion of ICUD increased from $9 \%$ in 2005 to $97 \%$ in 2015 . The ICUD group had a shorter operative time $(357$ vs. $400 \mathrm{~min}$, $\mathrm{P}<0.001)$, lower estimated blood loss (300 vs. $350 \mathrm{~mL}$, $\mathrm{P}<0.001)$, and lower blood transfusion rate $(5 \%$ vs. $13 \%$, $\mathrm{P}<0.001)$. The incidence of major complications in the ICUD group was $25 \%$ in 2005 , but decreased significantly to $6 \%$ in 2015 . This incidence remained stable in the ECUD group (13\% in 2005 and 14\% in 2015). Azzouni 
Table 2 Postoperative complications of 48 patients

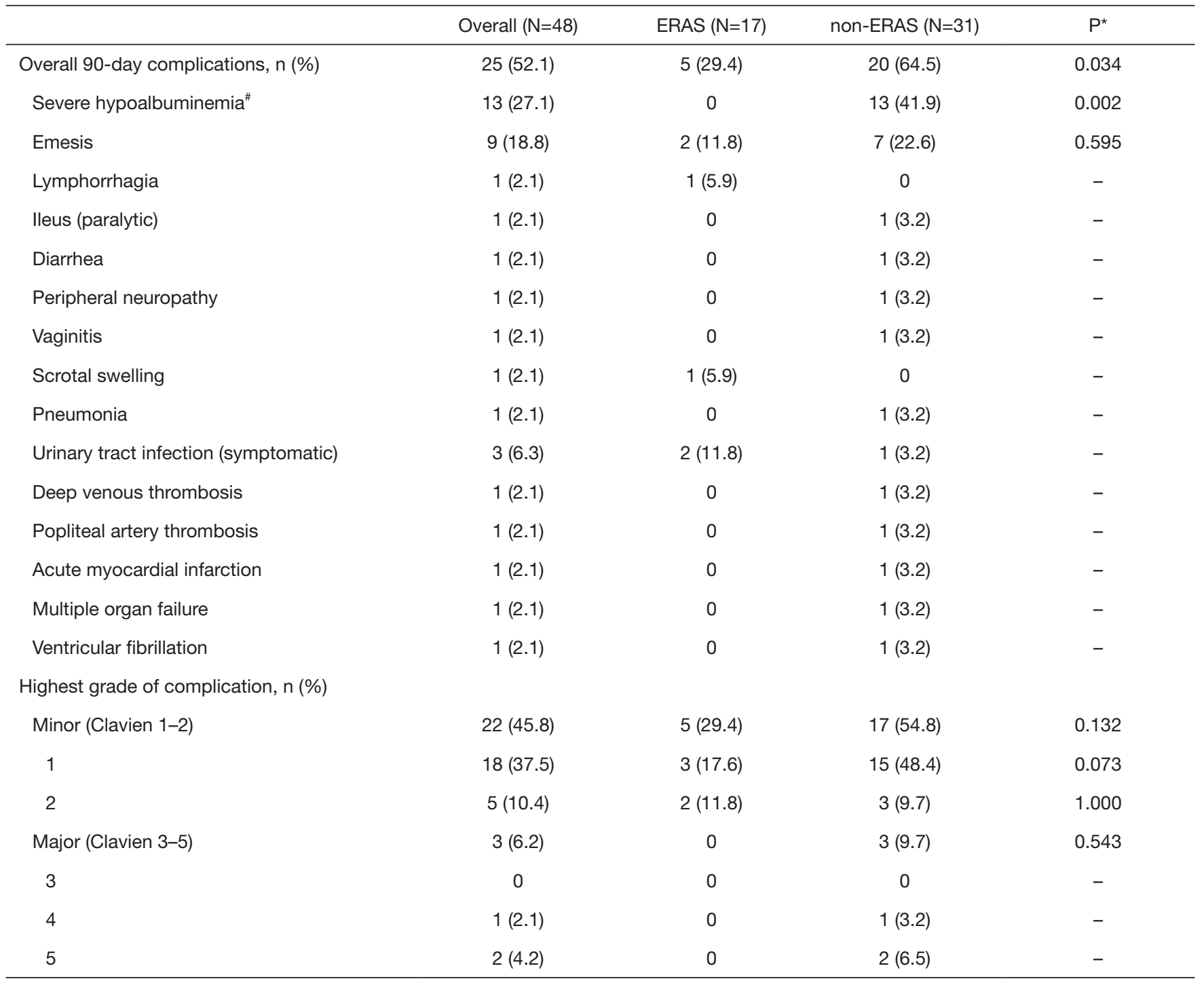

*comparison between ERAS and non-ERAS group; " serum albumin <25 g/L.

et al. (12) reported 90-day overall and major complication rates of intracorporeal urinary diversion of $81 \%$ and $19 \%$, respectively. Kanno et al. (13) compared the results of LRC with ICIC (72 cases) and extracorporeal ileal conduit (72 cases). The ICIC group had a longer operative time (676 vs. $616 \mathrm{~min}, \mathrm{P}=0.002)$, fewer wound-related complications ( $6 \%$ vs. $18 \%, \mathrm{P}=0.039$ ), and a shorter time to regular oral food intake ( 4 vs. 5 days, $\mathrm{P}=0.014$ ). According to a limited number of studies, the time to flatus and oral intake were shorter in ICIC/ICUD than in extracorporeal approach (13-15).

In our study, the time to flatus was two days and the time to liquid diet was three days. Operative time and urinary diversion time were 320 and $135 \mathrm{~min}$, respectively. Estimated blood loss was $200 \mathrm{~mL}$, and six patients $(12.5 \%)$ required intraoperative blood transfusion. These six patients belonged to the first half of the entire cohort, indicating that a learning curve that influences the transfusion rate may exist. The 90-day complication rate was $52.1 \%$. Minor complications were more common, with only $6.3 \%$ of patients developing major complications. The complication rate, operative time, and time to oral intake in our study were not inferior to those reported in previous studies (11-13).

ERAS protocols have gained significant attention in 
Table 3 Pathologic results of 48 patients

\begin{tabular}{|c|c|c|c|c|}
\hline & Overall $(\mathrm{N}=48)$ & ERAS $(\mathrm{N}=17)$ & non-ERAS $(\mathrm{N}=31)$ & $P^{*}$ \\
\hline $\mathrm{Ta}$ & $4(8.3)$ & $2(11.8)$ & $2(6.5)$ & \\
\hline Tis & $1(2.1)$ & 0 & $1(3.2)$ & \\
\hline $\mathrm{T} 1$ & $12(25.0)$ & $5(29.4)$ & $7(22.6)$ & \\
\hline T3 & $11(22.9)$ & $2(11.8)$ & $9(29.0)$ & \\
\hline $\mathrm{T} 4 \mathrm{a}$ & $8(16.7)$ & $2(11.8)$ & $6(19.4)$ & \\
\hline Pathologic stage (pNO-N3), n (\%) & & & & 0.689 \\
\hline NO & $29(60.4)$ & $12(70.6)$ & $17(54.8)$ & \\
\hline N3 & $7(14.6)$ & $1(5.9)$ & $6(19.4)$ & \\
\hline Grade of urothelial carcinoma, $n(\%)$ & & & & 1.000 \\
\hline Low grade & $3(6.3)$ & $1(5.9)$ & $2(6.5)$ & \\
\hline High grade & $42(87.5)$ & $15(88.2)$ & $27(87.1)$ & \\
\hline Lymph node yield (mean \pm SD) & $17.6 \pm 9.4$ & $13.9 \pm 6.5$ & $19.5 \pm 10.2$ & 0.052 \\
\hline Positive surgical margin, $\mathrm{n}(\%)$ & $4(8.3)$ & $1(5.9)$ & $3(9.7)$ & 1.000 \\
\hline
\end{tabular}

${ }^{*}$ comparison between ERAS and non-ERAS group.

Table 4 Morphology and function of upper urinary tract

\begin{tabular}{|c|c|c|c|c|c|c|}
\hline Grade, n (\%) & \multicolumn{3}{|c|}{ Hydronephrosis $(36 / 48)$} & \multicolumn{3}{|c|}{ Compromised renal function (36/48) } \\
\hline None & $26(72.2)$ & $30(83.3)$ & 0.317 & $19(52.8)$ & $19(52.8)$ & 0.940 \\
\hline Mild & $7(19.4)$ & $3(8.3)$ & & $12(33.3)$ & $13(36.1)$ & \\
\hline Moderate & $2(5.6)$ & $1(2.8)$ & & $5(13.9)$ & $3(8.3)$ & \\
\hline
\end{tabular}

recent years. A large-scale study (16) from the United Kingdom indicated that ERAS was associated with a shorter length of stay ( 8 vs. 18 days, $\mathrm{P}<0.001)$ and lower readmission rate $(15 \%$ vs. $25 \%, \mathrm{P}=0.04)$. A multiinstitutional, randomized controlled trial (17) conducted in China showed that ERAS was associated with faster recovery of bowel movement ( 88 vs. 100 hours, $\mathrm{P}=0.01$ ) and ambulation (64 vs. 72 hours, $\mathrm{P}=0.047$ ) when compared with the non-ERAS group. In the ERAS group of our research, the time to flatus ( 2 vs. 3 days, $\mathrm{P}=0.016$ ) and liquid diet ( 2 vs. 4 days, $\mathrm{P}<0.001)$ were both significantly shorter than the non-ERAS group. Length of stay also seemed to be shorter in the ERAS group, although the difference was not statistically significant ( 8 vs. 11 days, $\mathrm{P}=0.138$ ). ERAS patients experienced fewer complications (29.4\% vs. $64.5 \%, \mathrm{P}=0.034)$. Severe hypoalbuminemia incidence was significantly different, with an occurrence in nearly $41.9 \%$ of non-ERAS patients and zero ERAS patients. The main reason for postoperative hypoalbuminemia was attributed to transcapillary escape, because vascular 
permeability increased due to surgery-related inflammatory response $(18,19)$. Therefore, the implementation of ERAS, which aimed to reduce the stress response perioperatively, might have a major impact on reducing postoperative hypoalbuminemia.

Lymph node yield and PSM are two of the most important oncologic parameters of RC. Results from the Surveillance, Epidemiology, and End Results database showed that patients who had at least 10-14 lymph nodes removed had a better survival rate than those who underwent limited lymphadenectomy (20). PSM was mainly influenced by pathologic stage and surgeon volume. In high-volume academic centers, PSM rate varied from $2 \%$ to $10 \%$ (21). IRCC data presented a PSM rate of $6.8 \%$ (22). In our series, the lymph node yield was 17.6 \pm 9.4 . PSM was identified in four patients $(8.3 \%)$, including three cases of pT3 disease and one case of pT4 disease. These results demonstrated the oncologic quality of our technique.

In most studies, ICUD was accomplished using a roboticassisted approach. However, in China and other developing countries, the standard procedures for radical cystectomy are still open RC and LRC. The implementation of RARC is limited due to its expensive price, thus it will take a long time before RARC can replace open RC and LRC. Therefore, we have made an effort to modify the Bricker ileal conduit to reduce the difficulty of performing ICIC laparoscopically.

LRC with mICIC was completed through a minimally invasive method, which resulted in a smaller wound and reduced pain. When combined with the ERAS protocols, patients recovered bowel function faster and had fewer complications. During the surgery, the mesentery was transilluminated to maximally preserve the blood supply, which was especially important to allow isolation of a $15-\mathrm{cm}$ ileum segment with a narrow mesentery. An extraperitoneal tunnel was created, through which the distal end of the efferent loop was pulled out and fixed on the abdominal wall. With the efferent loop anchored in the extraperitoneal tunnel, the end-to-end reflux ureterointestinal anastomosis was performed directly and individually. In Wallace procedure, the two ureters were united before the ureterointestinal anastomosis. However, tumor recurrence, inflammation, or other diseases of the unilateral ureter, which could lead to obstruction, might also affect the contralateral ureter. Therefore, we omitted this step to avoid the mutual influence of bilateral ureters, and also reduce operative time as well. Finally, the distal ureters, efferent loop, and ureterointestinal anastomosis were totally retroperitonealized, thus avoiding the possibility of herniating the small bowel lateral to the conduit, reducing the incidence of urinary leak and secondary infection, and facilitating to handle subsequent complications.

Our study has several limitations. This was a retrospective study with a limited number of patients, who were not strictly randomized, and the follow-up time was relatively short.

\section{Conclusions}

The complication rate and operative time of the mICIC were acceptable. Clinical outcomes can be optimized with ERAS pathway.

\section{Acknowledgments}

We would like to thank Editage (www.editage.cn) for English language editing.

Funding: None.

\section{Footnote}

Reporting Checklist: The authors have completed the STROBE reporting checklist. Available at http://dx.doi. org/10.21037/tau-20-1515

Peer Review File: Available at http://dx.doi.org/10.21037/ tau-20-1515

Data Sharing Statement: Available at http://dx.doi. org/10.21037/tau-20-1515

Conflicts of Interest: All authors have completed the ICMJE uniform disclosure form (available at http://dx.doi. org/10.21037/tau-20-1515). The authors have no conflicts of interest to declare.

Ethical Statement: The authors are accountable for all aspects of the work in ensuring that questions related to the accuracy or integrity of any part of the work are appropriately investigated and resolved. The study was conducted in accordance with the Declaration of Helsinki (as revised in 2013). The study was approved by the Institutional Review Board of Beijing Chaoyang Hospital, Capital Medical University (NO.: 2020-Ke-546) and informed consent for this retrospective analysis was waived. 
Open Access Statement: This is an Open Access article distributed in accordance with the Creative Commons Attribution-NonCommercial-NoDerivs 4.0 International License (CC BY-NC-ND 4.0), which permits the noncommercial replication and distribution of the article with the strict proviso that no changes or edits are made and the original work is properly cited (including links to both the formal publication through the relevant DOI and the license). See: https://creativecommons.org/licenses/by-nc-nd/4.0/.

\section{References}

1. Lin T, Fan X, Zhang C, et al. A prospective randomised controlled trial of laparoscopic vs open radical cystectomy for bladder cancer: perioperative and oncologic outcomes with 5-year follow-up. Br J Cancer 2014;110:842-9.

2. Khan MS, Gan C, Ahmed K, et al. A Single-centre early phase randomised controlled three-arm trial of open, robotic, and laparoscopic radical cystectomy (CORAL). Eur Urol 2016;69:613-21.

3. Khan MS, Omar K, Ahmed K, et al. Long-term oncological outcomes from an early phase randomised controlled three-arm trial of open, robotic, and laparoscopic radical cystectomy (CORAL). Eur Urol 2020;77:110-8.

4. Ahmed K, Khan SA, Hayn MH, et al. Analysis of intracorporeal compared with extracorporeal urinary diversion after robot-assisted radical cystectomy: Results from the International Robotic Cystectomy Consortium. Eur Urol 2014;65:340-7.

5. Kehlet H. Multimodal approach to control postoperative pathophysiology and rehabilitation. Br J Anaesth 1997;78:606-17.

6. Collins JW, Patel H, Adding C, et al. Enhanced Recovery After Robot-assisted Radical Cystectomy: EAU Robotic Urology Section Scientific Working Group Consensus View. Eur Urol 2016;70:649-60.

7. Ni XF, Jia D, Chen Y, et al. Is the enhanced recovery after surgery (ERAS) program effective and safe in laparoscopic colorectal cancer surgery? A meta-analysis of randomized controlled trials. J Gastrointest Surg 2019;23:1502-12.

8. Lee Y, Yu J, Doumouras AG, et al. Enhanced recovery after surgery (ERAS) versus standard recovery for elective gastric cancer surgery: A meta-analysis of randomized controlled trials. Surg Oncol 2020;32:75-87.

9. Xing NZ, Song LM, Niu YN, et al. A novel technique of ureterointestinal anastomosis for urinary diversion.

Zhonghua Yi Xue Za Zhi 2012;92:114-6.

10. Gill IS, Fergany A, Klein EA, et al. Laparoscopic radical cystoprostatectomy with ileal conduit performed completely intracorporeally: the initial 2 cases. Urology 2000;56:26-9.

11. Hussein AA, May PR, Jing Z, et al. Outcomes of intracorporeal urinary diversion after robotassisted radical cystectomy: Results from the International Robotic Cystectomy Consortium. J Urol 2018;199:1302-11.

12. Azzouni FS, Din R, Rehman S, et al. The first 100 consecutive, robot-assisted, intracorporeal ileal conduits: Evolution of technique and 90-day outcomes. Eur Urol 2013;63:637-43.

13. Kanno T, Inoue T, Kawakita M, et al. Perioperative and oncological outcomes of laparoscopic radical cystectomy with intracorporeal versus extracorporeal ileal conduit: A matched-pair comparison in a multicenter cohort in Japan. Int J Urol 2020;27:559-65.

14. Shim JS, Kwon TG, Rha KH, et al. Do patients benefit from total intracorporeal robotic radical cystectomy?: A comparative analysis with extracorporeal robotic radical cystectomy from a Korean multicenter study. Investig Clin Urol 2020;61:11-8.

15. Wang MS, He QB, Yang FY, et al. A retrospective study comparing surgical and early oncological outcomes between intracorporeal and extracorporeal ileal conduit after laparoscopic radical cystectomy from a single center. Chin Med J 2018;131:784-9.

16. Pang KH, Groves R, Venugopal S, et al. Prospective Implementation of Enhanced Recovery After Surgery Protocols to Radical Cystectomy. Eur Urol 2018;73:363-71.

17. Lin TX, Li KW, Liu H, et al. Enhanced recovery after surgery for radical cystectomy with ileal urinary diversion: a multi-institutional, randomized, controlled trial from the Chinese bladder cancer consortium. World J Urol 2018;36:41-50.

18. Fleck A, Raines G, Hawker F, et al. Increased vascular permeability: A major cause of hypoalbuminaemia in disease and injury. Lancet 1985;1:781-4.

19. Smeets HJ, Kievit J, Dulfer FT, et al. Analysis of postoperative hypalbuminaemia: A clinical study. Int Surg 1994;79:152-7.

20. Konety BR, Joslyn SA, O Donnell MA. Extent of pelvic lymphadenectomy and its impact on outcome in patients diagnosed with bladder cancer: Analysis of data from the Surveillance, Epidemiology and End Results Program data 
base. J Urol 2003;169:946-50.

21. Skinner EC, Stein JP, Skinner DG. Surgical benchmarks for the treatment of invasive bladder cancer. Urol Oncol 2007;25:66-71.

Cite this article as: Wei $\mathrm{H}$, Wang $M$, Wasilijiang W, Wang W, Guan X, Zhou X, Song L, Xing N, Niu Y. Modified ileal conduit intracorporeally accomplished following laparoscopic radical cystectomy with enhanced recovery protocols: experience with 48 cases. Transl Androl Urol 2021;10(4):15961606. doi: 10.21037/tau-20-1515
22. Hellenthal NJ, Hussain A, Andrews PE, et al. Surgical margin status after robot assisted radical cystectomy: Results from the International Robotic Cystectomy Consortium. J Urol 2010;184:87-91. 